MATHEMATICS OF COMPUTATION

Volume 66, Number 217, January 1997, Pages 105-124

S 0025-5718(97)00795-3

\title{
AN ANALYSIS OF A CELL-VERTEX FINITE VOLUME METHOD FOR A PARABOLIC CONVECTION-DIFFUSION PROBLEM
}

\author{
WEN GUO AND MARTIN STYNES
}

\begin{abstract}
We examine a cell-vertex finite volume method which is applied to a model parabolic convection-diffusion problem. By using techniques from finite element analysis, local errors away from all layers are obtained in a seminorm that is related to, but weaker than, the $L^{2}$ norm.
\end{abstract}

\section{INTRODUCTION}

The cell-vertex finite volume method is a commonly used discretization scheme for conservation laws. It has been highly successful in modelling flows in aerodynamics. Since the method fits very naturally with convection problems, it has advantageous properties for convection-diffusion problems. Nevertheless, all analyses for cell-vertex methods have been carried out either for pure convection problems (see, e.g., Morton and Süli [10], Süli [15, 16] and Morton and Stynes [9]), or for convection-diffusion two-point boundary value problems (see, e.g., Mackenzie and Morton [8] and Morton and Stynes [9]). So far, there has been no similar analysis for a parabolic convection-diffusion problem in the literature.

In this paper, we examine a cell-vertex finite volume method when applied to the following model time-dependent convection-diffusion problem:

$$
\begin{aligned}
& L u(x, t) \equiv-\varepsilon u_{x x}+a u_{x}+b u+r u_{t}=f(x, t) \quad \forall(x, t) \in \Omega, \\
& u(0, t)=u(1, t)=0 \quad \text { for } \quad 0<t \leq T, \\
& u(x, 0)=u^{0}(x) \quad \text { for } \quad 0 \leq x \leq 1
\end{aligned}
$$

where $0<\varepsilon \ll a$ and $\Omega=(0,1) \times(0, T]$. For simplicity, we assume that $a, b$ and $r$ are constants with

$$
a>0, \quad b>0 \text { and } \quad r>0 .
$$

We also assume that $f \in L_{2}(\Omega)$ and $u^{0} \in L_{2}(0,1)$.

The conditions (1.1) - (1.4) define a time-dependent convection-diffusion problem. Problems of this type arise, for example, in the modelling of steady and unsteady viscous flow problems with large Reynolds numbers (see Peaceman and Rachford [13] and Van Dyke [17]), convective heat transport problems with large

Received by the editor August 23, 1993 and, in revised form, February 22, 1995 and January 26, 1996.

1991 Mathematics Subject Classification. Primary 65M60, 65M12; Secondary 76M25.

(C)1997 American Mathematical Society 
Peclet numbers (see Jakob [6]), oil reservoir simulation (see Ewing [4]), radioactive corrosion in the water cycles of an atomic reactor, adsorption processes in gas pipelines, spread of medicaments with the blood circulation or of plumes of poisonous industrial wastes in river systems (see Baumert et al. [2]), petroleum reservoir mechanics (see Price and Varga [14]) and electromagnetic field problems in moving media (see Hahn [5]). In (1.1), $\varepsilon$ is a diffusion coefficient and the function $a$ is a flow rate.

The differential operator in (1.1) is parabolic, but for small $\varepsilon$ its behavior might be considered as mixed parabolic-hyperbolic. The solution $u(x, t)$ will in general vary rapidly in a layer region of width $O(\varepsilon \ln (1 / \varepsilon))$ at boundary $x=1$, even for smooth initial-boundary data. This layer region is called a boundary layer. The boundary layer phenomenon has been discussed by many authors since Prandtl's original work in 1905; see, e.g., Vishik and Lyusternik [18], Eckhaus and de Jager [3], Nayfeh [11] and O'Malley [12]. It causes serious difficulties when solving (1.1) - (1.4) numerically.

An outline of the paper is as follows. In $\S 2$ we describe the cell-vertex method for (1.1) - (1.4) and reformulate it as a finite element method. Section 3 is devoted to the derivation of a discrete Gårding inequality that guarantees the existence and uniqueness of the finite volume solution. Local errors in the $l_{2}$ seminorm (defined in $\S 3$ ) are analyzed in $\S 4$. (We note that, when restricted to certain piecewise bilinear trial spaces, this seminorm becomes a norm.) Our analysis indicates that on a general tensor product mesh, the method is first-order accurate away from all layers, in the $l_{2}$ seminorm. We can sharpen this result to local second-order accuracy in $l_{2}$, if either $\varepsilon$ is very small compared to the mesh diameter or the mesh is locally almost uniform.

Throughout the paper, we shall use $C$ to denote a generic positive constant which is independent of $\varepsilon$ and of any mesh used.

\section{Description of the CELl-Vertex SCheme}

To discretize (1.1) - (1.4), we first define a partition of $\Omega$ as follows. For any pair of positive integers $N$ and $M$, we consider the arbitrary tensor product grid

$$
\begin{aligned}
\Omega^{h}=\left\{\left(x_{i}, t_{j}\right) \in \Omega: 0\right. & =x_{0}<x_{1}<\cdots<x_{N}=1, \\
& \left.=t_{0}<t_{1}<\cdots<t_{M}=T\right\}
\end{aligned}
$$

with $h_{i}=x_{i}-x_{i-1}, k_{j}=t_{j}-t_{j-1}$ and $h=\max _{i, j}\left\{h_{i}, k_{j}\right\}$. Define the "finite volume" or "cell" $K_{i, j}$ by

$$
K_{i, j}=\left(x_{i-1}, x_{i}\right) \times\left(t_{j-1}, t_{j}\right), \quad \text { for } i=1, \ldots, N \text { and } j=1, \ldots, M .
$$

In the finite volume context, the discretization of (1.1) is performed on each cell. The basic idea is to integrate (1.1) over a cell so that the convection and diffusion terms are converted into line integrals of normal fluxes along the cell edges, and then use the trapezoidal rule to approximate the integrals. Thus, letting $u^{h}$ denote 
the computed solution, for cell $K_{i, j}$ we set

$(2.1)$

$$
\begin{array}{rl}
\iint_{K_{i, j}} & f(x, t) d x d t \\
= & -\frac{\varepsilon k_{j}}{2}\left(u_{x}^{h}\left(x_{i}, t_{j}\right)-u_{x}^{h}\left(x_{i-1}, t_{j}\right)+u_{x}^{h}\left(x_{i}, t_{j-1}\right)-u_{x}^{h}\left(x_{i-1}, t_{j-1}\right)\right) \\
& +\frac{a k_{j}}{2}\left(u^{h}\left(x_{i}, t_{j}\right)-u^{h}\left(x_{i-1}, t_{j}\right)+u^{h}\left(x_{i}, t_{j-1}\right)-u^{h}\left(x_{i-1}, t_{j-1}\right)\right) \\
& +\frac{r h_{i}}{2}\left(u^{h}\left(x_{i}, t_{j}\right)-u^{h}\left(x_{i}, t_{j-1}\right)+u^{h}\left(x_{i-1}, t_{j}\right)-u^{h}\left(x_{i-1}, t_{j-1}\right)\right) \\
& +\frac{b h_{i} k_{j}}{4}\left(u^{h}\left(x_{i}, t_{j}\right)+u^{h}\left(x_{i-1}, t_{j}\right)+u^{h}\left(x_{i}, t_{j-1}\right)+u^{h}\left(x_{i-1}, t_{j-1}\right)\right) .
\end{array}
$$

With the approximation $u^{h}(x, t)$ parameterized by its values at the vertices, this still leaves two problems to be solved. First, how do we define $u_{x}^{h}$ at the nodes? There are several ways in which this may be done, but we consider here the so-called Method A in Mackenzie and Morton [8]. That is, we define

$$
u_{x}^{h}\left(x_{i}, t_{j}\right)=\frac{1}{h_{i}+h_{i+1}}\left(u^{h}\left(x_{i+1}, t_{j}\right)-u^{h}\left(x_{i-1}, t_{j}\right)\right),
$$

for $i=1, \ldots, N-1$, and

$$
u_{x}^{h}\left(0, t_{j}\right)=\frac{2}{h_{1}}\left(u^{h}\left(x_{1}, t_{j}\right)-u^{h}\left(0, t_{j}\right)\right)-u_{x}^{h}\left(x_{1}, t_{j}\right) .
$$

Similarly to $(2.3)$, one can define $u_{x}^{h}\left(1, t_{j}\right)$. This solves the first problem.

The second difficulty is as follows. If we perform the discretization (2.1) on all cells, we will have a system of $N M$ equations in $(N-1) M$ unknowns, since $u^{h}(x, t)$ will be prescribed on three sides of $\Omega$ by (1.2) and (1.3). We have $M$ equations too many. To obtain an exact match, we choose upwind control volumes, that is, each nodal unknown is associated with the cell upwind of it. We do this by discarding the equations associated with $K_{N, j}$ for $j=1, \ldots, M$. We then obtain a system of equations $(2.1)-(2.3)$, for $i=1, \ldots, N-1$ and $j=1, \ldots, M$, which has exactly the same number of unknowns as that of equations. The second problem has been eliminated.

Finite volume methods are often interpreted as finite difference methods. This is reflected in the finite difference techniques used to analyze such schemes. For a scheme such as $(2.1)-(2.3)$, which does not satisfy a discrete maximum principle, it is difficult to obtain a satisfactory finite difference analysis. Instead, we observe that the cell-vertex formulation of the finite volume method has a natural interpretation as a Petrov-Galerkin finite element method. The finite element framework then affords the possibility of applying finite element techniques to estimate errors in the finite volume method; see $[10,15,16,9]$.

To reformulate the cell-vertex finite volume scheme $(2.1)-(2.3)$ as a finite element method, we first define our trial and test spaces. Set

$$
\begin{aligned}
\mathcal{U}_{0}^{h}=\left\{v \in H^{1}(\Omega) \cap C(\bar{\Omega}):\right. & v(0, t)=v(1, t)=0 \text { for } t \in(0, T], \\
& v \text { is bilinear on each cell } K\}, \\
\mathcal{M}^{h}=\left\{p \in L^{2}(\Omega):\right. & p \text { is constant on each cell } K, \\
& \left.p \equiv 0 \text { on cells } K_{N, j} \text { for } j=1, \ldots, M\right\} .
\end{aligned}
$$


In order to simplify the presentation, we introduce the averaging operators $\mu, \mu_{x}$ and $\mu_{t}$, for $i=1, \ldots, N$ and $j=1, \ldots, M$,

$$
\begin{aligned}
\mu w_{i, j} & =\frac{1}{h_{i} k_{j}} \iint_{K_{i, j}} w(x, t) d x d t, \\
\mu_{x} w_{i, j} & =\frac{1}{h_{i}} \int_{x_{i-1}}^{x_{i}} w\left(x, t_{j}\right) d x, \\
\mu_{t} w_{i, j} & =\frac{1}{k_{j}} \int_{t_{j-1}}^{t_{j}} w\left(x_{i}, t\right) d t,
\end{aligned}
$$

for all $w(x, t)$ for which the right-hand side is defined.

Remark 2.1. One can easily verify that for each $v \in \mathcal{U}_{0}^{h}$ and for $i=1, \ldots, N$ and $j=1, \ldots, M$,

$$
\begin{aligned}
\mu v_{i, j} & =\frac{1}{4}\left(v_{i-1, j}+v_{i, j}+v_{i-1, j-1}+v_{i, j-1}\right) \\
& =\frac{1}{2}\left(\mu_{x} v_{i, j}+\mu_{x} v_{i, j-1}\right) \\
& =\frac{1}{2}\left(\mu_{t} v_{i, j}+\mu_{t} v_{i-1, j}\right), \\
\mu\left(v_{x}\right)_{i, j} & =\frac{1}{h_{i}}\left(\mu_{t} v_{i, j}-\mu_{t} v_{i-1, j}\right), \\
\mu\left(v_{t}\right)_{i, j} & =\frac{1}{k_{j}}\left(\mu_{x} v_{i, j}-\mu_{x} v_{i, j-1}\right),
\end{aligned}
$$

where $v_{i, j}$ denotes $v\left(x_{i}, t_{j}\right)$.

Now the cell-vertex finite volume approximation is defined as follows: find $u^{h} \in$ $\mathcal{U}_{0}^{h}$ satisfying

$$
\begin{gathered}
\hat{B}\left(u^{h}, p\right)=(f, p) \quad \forall p \in \mathcal{M}^{h}, \\
\left\langle u^{h}(\cdot, 0), p^{+}\right\rangle=\left\langle u^{0}, p^{+}\right\rangle \quad \forall p \in \mathcal{M}^{h},
\end{gathered}
$$

where $(\cdot, \cdot)$ and $\langle\cdot, \cdot\rangle$ are the usual $L^{2}(\Omega)$ and $L^{2}(0,1)$ inner products,

$$
p^{+}(x)=\lim _{t \rightarrow 0^{+}} p(x, t)
$$

and we set for any $(v, p) \in H^{1}(\Omega) \times \mathcal{M}^{h}$,

$$
\begin{aligned}
\hat{B}(v, p)= & -\varepsilon \sum_{j=1}^{M} \sum_{i=1}^{N-1} k_{j} \mu p_{i, j}\left\{\hat{\mu}_{t}\left(v_{x}\right)_{i, j}-\hat{\mu}_{t}\left(v_{x}\right)_{i-1, j}\right\} \\
& +\left(a v_{x}+r v_{t}+b v, p\right) .
\end{aligned}
$$

Here we define, for $j=1, \ldots, M$ and $v \in C(\Omega)$,

$$
\hat{\mu}_{t}\left(v_{x}\right)_{i, j}= \begin{cases}\frac{2}{h_{i}+h_{i+1}}\left(\mu v_{i+1, j}-\mu v_{i, j}\right) & \text { if } i=1, \ldots, N-1, \\ \frac{2}{h_{1}} \mu_{t} v_{1, j}-\hat{\mu}_{t}\left(v_{x}\right)_{1, j} & \text { if } i=0 .\end{cases}
$$

Remark 2.2. For the discretization of the diffusion term in $\hat{B}(v, p)$, we do not need nodal values of $v_{x}$, just its integral along two sides $x=x_{i-1}$ and $x=x_{i}$ of $K_{i, j}$. For $i=1, \ldots, N-1, \hat{\mu}_{t}\left(v_{x}\right)_{i, j}$ is defined by associating $\mu v_{i, j}$ with the cell center, 
then taking the obvious divided difference. For $i=0$, we define $\hat{\mu}_{t}\left(v_{x}\right)_{i, j}$ by an extrapolation. It is easy to check that $(2.1)-(2.3)$ is equivalent to $(2.9)-(2.12)$.

In the next section, we shall demonstrate existence and uniqueness of the finite volume solution.

\section{Stability And CONVERgence}

We begin our analysis of the cell-vertex finite volume scheme $(2.9)-(2.12)$ by establishing the stability of the method in some appropriate mesh-dependent norms, which in turn implies the existence and uniqueness of the finite volume solution $u^{h}$.

We introduce the following mesh-dependent norms:

$$
\begin{aligned}
& |v|_{l_{2}\left(\Omega^{h}\right)}=\left\{\sum_{j=1}^{M} \sum_{i=1}^{N-1} h_{i} k_{j}\left|\mu v_{i, j}\right|^{2}\right\}^{1 / 2}, \\
& |v|_{l_{2}\left(\partial_{+} \Omega^{h}\right)}=\left\{\sum_{j=1}^{M} k_{j}\left|\mu_{t} v_{N-1, j}\right|^{2}+\sum_{i=1}^{N-1} h_{i}\left|\mu_{x} v_{i, M}\right|^{2}\right\}^{1 / 2}, \\
& |v|_{l_{2}\left(\partial_{-} \Omega^{h}\right)}=\left\{\sum_{i=1}^{N-1} h_{i}\left|\mu_{x} v_{i, 0}\right|^{2}\right\}^{1 / 2}, \\
& \left|v_{x}\right|_{\hat{l}_{2}\left(\Omega^{h}\right)}=\left\{\sum_{j=1}^{M} \sum_{i=1}^{N-1} \frac{h_{i}+h_{i+1}}{2} k_{j}\left|\hat{\mu}_{t}\left(v_{x}\right)_{i, j}\right|^{2}+\frac{h_{1}}{4} \sum_{j=1}^{M} k_{j}\left|\hat{\mu}_{t}\left(v_{x}\right)_{0, j}\right|^{2}\right\}^{1 / 2},
\end{aligned}
$$

for all $v(x, t)$ for which the right-hand sides are defined.

Remark 3.1. We note that these norms are seminorms on $L^{2}(\Omega)$. If $|\cdot| l_{2_{2}\left(\Omega^{h}\right)}$ is restricted to the subspace of $\mathcal{U}_{0}^{h}$ defined by

$$
\left\{v \in \mathcal{U}_{0}^{h}: v(x, 0)=0 \text { for } 0 \leq x \leq 1\right\},
$$

then it is a norm. The first three of these seminorms are similar to those used in Süli [16]. The last seminorm is introduced here to deal with the diffusion term.

Define a projection $R: \mathcal{U}_{0}^{h} \rightarrow \mathcal{M}^{h}$ by

$$
R v= \begin{cases}\mu v_{i, j} & \text { on } K_{i, j}, \text { for } i=1, \ldots, N-1 \text { and } j=1, \ldots, M, \\ 0 & \text { otherwise. }\end{cases}
$$

The stability of the finite volume method $(2.9)-(2.12)$ is proved by the following discrete Gårding inequality.

Theorem 3.1. Assume that $\varepsilon \leq a\left(h_{N-1}+h_{N}\right)$. Then for each $v \in \mathcal{U}_{0}^{h}$,

$$
\begin{aligned}
\hat{B}(v, R v) \geq & \frac{\varepsilon}{2}\left|v_{x}\right|_{\hat{l}_{2}\left(\Omega^{h}\right)}^{2}+b|v|_{l_{2}\left(\Omega^{h}\right)}^{2} \\
& +\frac{1}{4} \min \{a, r\}|v|_{l_{2}\left(\partial_{+} \Omega^{h}\right)}^{2}-\frac{r}{2}|v|_{l_{2}\left(\partial_{-} \Omega^{h}\right)}^{2} .
\end{aligned}
$$


Proof. Recall the definition $(2.11)$ of $\hat{B}(\cdot, \cdot)$. For each $v \in \mathcal{U}_{0}^{h}$,

$$
\begin{aligned}
\hat{B}(v, R v)= & -\varepsilon \sum_{j=1}^{M} \sum_{i=1}^{N-1} k_{j} \mu v_{i, j}\left\{\hat{\mu}_{t}\left(v_{x}\right)_{i, j}-\hat{\mu}_{t}\left(v_{x}\right)_{i-1, j}\right\} \\
& +\left(a v_{x}+r v_{t}+b v, R v\right) \\
\equiv & I_{1}+I_{2} .
\end{aligned}
$$

First, by summation by parts,

$$
\begin{array}{r}
I_{1}=\varepsilon \sum_{j=1}^{M} k_{j}\left\{-\mu v_{N-1, j} \hat{\mu}_{t}\left(v_{x}\right)_{N-1, j}+\mu v_{1, j} \hat{\mu}_{t}\left(v_{x}\right)_{0, j}\right. \\
\left.+\sum_{i=1}^{N-2}\left(\mu v_{i+1, j}-\mu v_{i, j}\right) \hat{\mu}_{t}\left(v_{x}\right)_{i, j}\right\} .
\end{array}
$$

Now we have

$$
\begin{aligned}
-\mu v_{N-1, j} \hat{\mu}_{t}\left(v_{x}\right)_{N-1, j} & \\
= & \left(\mu v_{N, j}-\mu v_{N-1, j}\right) \hat{\mu}_{t}\left(v_{x}\right)_{N-1, j}-\mu v_{N, j} \hat{\mu}_{t}\left(v_{x}\right)_{N-1, j} \\
= & \frac{h_{N-1}+h_{N}}{2}\left|\hat{\mu}_{t}\left(v_{x}\right)_{N-1, j}\right|^{2}-\frac{1}{2} \mu_{t} v_{N-1, j} \hat{\mu}_{t}\left(v_{x}\right)_{N-1, j}, \\
& \quad \operatorname{using}(2.12),(2.6) \text { and } \mu_{t} v_{N, j}=0, \\
& \geq \frac{h_{N-1}+h_{N}}{4}\left|\hat{\mu}_{t}\left(v_{x}\right)_{N-1, j}\right|^{2}-\frac{1}{4\left(h_{N-1}+h_{N}\right)}\left|\mu_{t} v_{N-1, j}\right|^{2},
\end{aligned}
$$

by the arithmetic-geometric inequality

$$
2 \alpha \beta \leq \alpha^{2} / q+q \beta^{2}
$$

for all $q>0$ and all $\alpha$ and $\beta$. Similarly, using (2.6) and $\mu_{t} v_{0, j}=0$, we have

$$
\begin{aligned}
\mu v_{1, j} & \hat{\mu}_{t}\left(v_{x}\right)_{0, j} \\
& =\frac{1}{2} \mu_{t} v_{1, j} \hat{\mu}_{t}\left(v_{x}\right)_{0, j} \\
& =\frac{h_{1}}{4}\left(\hat{\mu}_{t}\left(v_{x}\right)_{0, j}+\hat{\mu}_{t}\left(v_{x}\right)_{1, j}\right) \hat{\mu}_{t}\left(v_{x}\right)_{0, j}, \quad \text { by }(2.12), \\
& \geq \frac{h_{1}}{8}\left(\left|\hat{\mu}_{t}\left(v_{x}\right)_{0, j}\right|^{2}-\left|\hat{\mu}_{t}\left(v_{x}\right)_{1, j}\right|^{2}\right) .
\end{aligned}
$$

Also from (2.12), for $i=1, \ldots, N-2$, we obtain

$$
\left(\mu v_{i+1, j}-\mu v_{i, j}\right) \hat{\mu}_{t}\left(v_{x}\right)_{i, j}=\frac{h_{i}+h_{i+1}}{2}\left|\hat{\mu}_{t}\left(v_{x}\right)_{i, j}\right|^{2} .
$$

Substituting (3.3) - (3.5) into (3.2), we get

$$
\begin{gathered}
I_{1} \geq \varepsilon \sum_{j=1}^{M} k_{j}\left\{\frac{h_{N-1}+h_{N}}{4}\left|\hat{\mu}_{t}\left(v_{x}\right)_{N-1, j}\right|^{2}-\frac{1}{4\left(h_{N-1}+h_{N}\right)}\left|\mu_{t} v_{N-1, j}\right|^{2}\right. \\
+\frac{h_{1}}{8}\left(\left|\hat{\mu}_{t}\left(v_{x}\right)_{0, j}\right|^{2}-\left|\hat{\mu}_{t}\left(v_{x}\right)_{1, j}\right|^{2}\right) \\
\left.+\sum_{i=1}^{N-2} \frac{h_{i}+h_{i+1}}{2}\left|\hat{\mu}_{t}\left(v_{x}\right)_{i, j}\right|^{2}\right\} .
\end{gathered}
$$


Next, we have

$$
\begin{aligned}
I_{2}= & \sum_{j=1}^{M} \sum_{i=1}^{N-1} h_{i} k_{j} \mu\left(a v_{x}+r v_{t}+b v\right)_{i, j} \mu v_{i, j} \\
= & \sum_{j=1}^{M} \sum_{i=1}^{N-1}\left\{a k_{j}\left(\mu_{t} v_{i, j}-\mu_{t} v_{i-1, j}\right)+r h_{i}\left(\mu_{x} v_{i, j}-\mu_{x} v_{i, j-1}\right)+b h_{i} k_{j} \mu v_{i, j}\right\} \mu v_{i, j}, \\
= & \frac{a}{2} \sum_{j=1}^{M} k_{j} \sum_{i=1}^{N-1}\left(\left|\mu_{t} v_{i, j}\right|^{2}-\left|\mu_{t} v_{i-1, j}\right|^{2}\right)+\frac{r}{2} \sum_{i=1}^{N-1} h_{i} \sum_{j=1}^{M}\left(\left|\mu_{x} v_{i, j}\right|^{2}-\left|\mu_{x} v_{i, j-1}\right|^{2}\right) \\
& +b \sum_{j=1}^{M} \sum_{i=1}^{N-1} h_{i} k_{j}\left|\mu v_{i, j}\right|^{2}, \quad \operatorname{using}(2.5) \text { and }(2.6), \\
& =\frac{a}{2} \sum_{j=1}^{M} k_{j}\left|\mu_{t} v_{N-1, j}\right|^{2}+\frac{r}{2} \sum_{i=1}^{N-1} h_{i}\left(\left|\mu_{x} v_{i, M}\right|^{2}-\left|\mu_{x} v_{i, 0}\right|^{2}\right) \\
& +b \sum_{j=1}^{M} \sum_{i=1}^{N-1} h_{i} k_{j}\left|\mu v_{i, j}\right|^{2},
\end{aligned}
$$

by telescoping and using $\mu_{t} v_{0, j}=0$, for $j=1, \ldots, M$.

Hence,

$$
\begin{aligned}
\hat{B}(v, R v)= & I_{1}+I_{2} \\
\geq & \frac{\varepsilon}{2}\left|v_{x}\right|_{\hat{l}_{2}\left(\Omega^{h}\right)}^{2}+\left(\frac{a}{2}-\frac{\varepsilon}{4\left(h_{N-1}+h_{N}\right)}\right) \sum_{j=1}^{M} k_{j}\left|\mu_{t} v_{N-1, j}\right|^{2} \\
& +\frac{r}{2} \sum_{i=1}^{N-1} h_{i}\left|\mu_{x} v_{i, M}\right|^{2}-\frac{r}{2}|v|_{l_{2}\left(\partial_{-} \Omega^{h}\right)}^{2}+b|v|_{l_{2}\left(\Omega^{h}\right)}^{2} .
\end{aligned}
$$

The desired result then follows from the assumption of the theorem.

As a corollary we obtain the following stability result.

Theorem 3.2. Assume that $\varepsilon \leq a\left(h_{N-1}+h_{N}\right)$. Then (2.9) - (2.12) has a unique solution $u^{h} \in \mathcal{U}_{0}^{h}$ and

$$
\begin{gathered}
\varepsilon\left|u_{x}^{h}\right|_{\hat{l}_{2}\left(\Omega^{h}\right)}^{2}+\left|u^{h}\right|_{l_{2}\left(\Omega^{h}\right)}^{2}+\left|u^{h}\right|_{l_{2}\left(\partial_{+} \Omega^{h}\right)}^{2} \\
\leq C\left\{|f|_{l_{2}\left(\Omega^{h}\right)}^{2}+\left|u^{0}\right|_{l_{2}\left(\partial_{-} \Omega^{h}\right)}^{2}\right\} .
\end{gathered}
$$

Here, $C$ depends only on $a, b$ and $r$.

Proof. As the existence of a unique solution follows from (3.8), because we are dealing with a norm in this situation (cf. Remark 3.1), we need only establish (3.8).

Taking $p=R u^{h}$ in (2.9) and using the arithmetic-geometric inequality, we obtain

$$
\begin{aligned}
\hat{B}\left(u^{h}, R u^{h}\right) & =\left(f, R u^{h}\right) \\
& \leq \frac{b}{2}\left|u^{h}\right|_{l_{2}\left(\Omega^{h}\right)}^{2}+\frac{1}{2 b}|f|_{l_{2}\left(\Omega^{h}\right)}^{2} .
\end{aligned}
$$


Now an appeal to Theorem 3.1 completes the proof.

Theorems 3.1 and 3.2 are in the spirit of results obtained in finite element analyses using standard "inf-sup" machinery; see, e.g., Babuška and Osborn [1] and the references therein.

As another corollary of Theorem 3.1 we have the following global error bound.

Theorem 3.3. Assume that $\varepsilon \leq a\left(h_{N-1}+h_{N}\right)$. Let $u^{I}$ be the interpolant from $\mathcal{U}_{0}^{h}$ to $u$. Then

$$
\begin{aligned}
\varepsilon\left|\mu_{t}\left(u_{x}\right)-\hat{\mu}_{t}\left(u_{x}^{h}\right)\right|_{l_{2}^{\circ}\left(\Omega^{h}\right)}^{2}+\left|u-u^{h}\right|_{l_{2}\left(\Omega^{h}\right)}^{2}+\left|u-u^{h}\right|_{l_{2}\left(\partial_{+} \Omega^{h}\right)}^{2} \\
\leq C\left\{\varepsilon\left|\mu_{t}\left(u_{x}\right)-\hat{\mu}_{t}\left(u_{x}^{I}\right)\right|_{l_{2}^{\circ}\left(\Omega^{h}\right)}^{2}+\left|a\left(u-u^{I}\right)_{x}+r\left(u-u^{I}\right)_{t}\right|_{l_{2}\left(\Omega^{h}\right)}^{2}\right. \\
\left.\quad+\left|u-u^{I}\right|_{l_{2}\left(\Omega^{h}\right)}^{2}+\left|u-u^{I}\right|_{l_{2}\left(\partial_{-} \Omega^{h}\right)}^{2}+\left|u-u^{I}\right|_{l_{2}\left(\partial_{+} \Omega^{h}\right)}^{2}\right\}
\end{aligned}
$$

where

$$
\begin{aligned}
\left|\mu_{t}\left(u_{x}\right)-\hat{\mu}_{t}\left(u_{x}^{h}\right)\right|_{l_{2}^{\circ}\left(\Omega^{h}\right)}^{2}= & \sum_{j=1}^{M} k_{j} \sum_{i=1}^{N-1} \frac{h_{i}+h_{i+1}}{2}\left|\mu_{t}\left(u_{x}\right)_{i, j}-\hat{\mu}_{t}\left(u_{x}^{h}\right)_{i, j}\right|^{2} \\
& +\frac{h_{1}}{4} \sum_{j=1}^{M} k_{j}\left|\mu_{t}\left(u_{x}\right)_{0, j}-\hat{\mu}_{t}\left(u_{x}^{h}\right)_{0, j}\right|^{2}
\end{aligned}
$$

and $\left|\mu_{t}\left(u_{x}\right)-\hat{\mu}_{t}\left(u_{x}^{I}\right)\right|_{l_{2}^{\circ}\left(\Omega^{h}\right)}^{2}$ is similarly defined.

Proof. Set

$$
\xi=u^{h}-u^{I}, \quad \eta=u-u^{I}
$$

Then

$$
u-u^{h}=\eta-\xi
$$

We begin by estimating $\xi$.

Applying Theorem 3.1, we obtain

$$
\begin{gathered}
\frac{\varepsilon}{2}\left|\xi_{x}\right|_{\hat{l}_{2}\left(\Omega^{h}\right)}^{2}+b|\xi|_{l_{2}\left(\Omega^{h}\right)}^{2}+\frac{1}{4} \min \{a, r\}|\xi|_{l_{2}\left(\partial_{+} \Omega^{h}\right)}^{2} \\
\leq \hat{B}(\xi, R \xi)+\frac{r}{2}|\xi|_{l_{2}\left(\partial_{-} \Omega^{h}\right)}^{2} .
\end{gathered}
$$

Set

$$
e_{i, j}=\mu_{t}\left(u_{x}\right)_{i, j}-\hat{\mu}_{t}\left(u_{x}^{I}\right)_{i, j} .
$$

From (1.1) - (1.3) and (2.9) - (2.12), we have

$$
\begin{aligned}
\hat{B}(\xi, R \xi)= & -\varepsilon \sum_{j=1}^{M} \sum_{i=1}^{N-1} k_{j} \mu \xi_{i, j}\left\{e_{i, j}-e_{i-1, j}\right\} \\
& +\left(a \eta_{x}+r \eta_{t}+b \eta, R \xi\right)
\end{aligned}
$$

and, by (2.10),

$$
|\xi|_{l_{2}\left(\partial_{-} \Omega^{h}\right)}^{2}=|\eta|_{l_{2}\left(\partial_{-} \Omega^{h}\right)}^{2} .
$$


We first estimate the term involving $\varepsilon$ :

$$
\begin{aligned}
& -\varepsilon \sum_{j=1}^{M} \sum_{i=1}^{N-1} k_{j} \mu \xi_{i, j}\left\{e_{i, j}-e_{i-1, j}\right\} \\
& =\varepsilon \sum_{j=1}^{M} k_{j}\left\{-\mu \xi_{N, j} e_{N-1, j}+\mu \xi_{1, j} e_{0, j}+\sum_{i=1}^{N-1}\left(\mu \xi_{i+1, j}-\mu \xi_{i, j}\right) e_{i, j}\right\}, \\
& \text { by summation by parts, } \\
& =\varepsilon \sum_{j=1}^{M} k_{j}\left\{-\frac{1}{2} \mu_{t} \xi_{N-1, j} e_{N-1, j}+\frac{1}{2} \mu_{t} \xi_{1, j} e_{0, j}\right. \\
& \left.+\sum_{i=1}^{N-1} \frac{h_{i}+h_{i+1}}{2} \hat{\mu}_{t}\left(\xi_{x}\right)_{i, j} e_{i, j}\right\} \\
& \text { by virtue of (2.12), (2.6) and } \mu_{t} \xi_{0, j}=\mu_{t} \xi_{N, j}=0, \\
& =\varepsilon \sum_{j=1}^{M} k_{j}\left\{-\frac{1}{2} \mu_{t} \xi_{N-1, j} e_{N-1, j}+\frac{h_{1}}{4}\left(\hat{\mu}_{t}\left(\xi_{x}\right)_{0, j}+\hat{\mu}_{t}\left(\xi_{x}\right)_{1, j}\right) e_{0, j}\right. \\
& \left.+\sum_{i=1}^{N-1} \frac{h_{i}+h_{i+1}}{2} \hat{\mu}_{t}\left(\xi_{x}\right)_{i, j} e_{i, j}\right\}, \quad \text { by }(2.12), \\
& \leq \varepsilon \sum_{j=1}^{M} k_{j}\left\{\frac{\min \{a, r\}}{8 \varepsilon}\left|\mu_{t} \xi_{N-1, j}\right|^{2}+C \varepsilon\left|e_{N-1, j}\right|^{2}\right. \\
& +\frac{h_{1}}{16}\left|\hat{\mu}_{t}\left(\xi_{x}\right)_{0, j}\right|^{2}+\frac{h_{1}}{16}\left|\hat{\mu}_{t}\left(\xi_{x}\right)_{1, j}\right|^{2}+C h_{1}\left|e_{0, j}\right|^{2} \\
& \left.+\frac{1}{8} \sum_{i=1}^{N-1} \frac{h_{i}+h_{i+1}}{2}\left|\hat{\mu}_{t}\left(\xi_{x}\right)_{i, j}\right|^{2}+2 \sum_{i=1}^{N-1} \frac{h_{i}+h_{i+1}}{2}\left|e_{i, j}\right|^{2}\right\} \\
& \leq \frac{1}{8} \min \{a, r\}|\xi|_{l_{2}\left(\partial_{+} \Omega^{h}\right)}^{2}+\frac{\varepsilon}{4}\left|\xi_{x}\right|_{\hat{l}_{2}\left(\Omega^{h}\right)}^{2}+C \varepsilon\left|\mu_{t}\left(u_{x}\right)-\hat{\mu}_{t}\left(u_{x}^{I}\right)\right|_{l_{2}^{\circ}\left(\Omega^{h}\right)}^{2},
\end{aligned}
$$

using $\varepsilon \leq a\left(h_{N-1}+h_{N}\right)$.

As regards the other term in (3.12), we have

$$
\left(a \eta_{x}+r \eta_{t}+b \eta, R \xi\right) \leq \frac{b}{2}|\xi|_{l_{2}\left(\Omega^{h}\right)}^{2}+C\left|a \eta_{x}+r \eta_{t}+b \eta\right|_{l_{2}\left(\Omega^{h}\right)}^{2} .
$$

Thus, from (3.10), (3.12) - (3.15), it follows that

$$
\begin{aligned}
& \frac{\varepsilon}{4}\left|\xi_{x}\right|_{\hat{l}_{2}\left(\Omega^{h}\right)}^{2}+\frac{b}{2}|\xi|_{l_{2}\left(\Omega^{h}\right)}^{2}+\frac{1}{8} \min \{a, r\}|\xi|_{l_{2}\left(\partial_{+} \Omega^{h}\right)}^{2} \\
& \quad \leq C\left\{\varepsilon\left|\mu_{t}\left(u_{x}\right)-\hat{\mu}_{t}\left(u_{x}^{I}\right)\right|_{l_{2}^{\circ}\left(\Omega^{h}\right)}^{2}+\left|a \eta_{x}+r \eta_{t}+b \eta\right|_{l_{2}\left(\Omega^{h}\right)}^{2}+|\eta|_{l_{2}\left(\partial_{-} \Omega^{h}\right)}^{2}\right\} .
\end{aligned}
$$

This, together with the triangle inequality, yields the desired result.

\section{LOCAL ERROR ANALYSIS}

In this section we present local convergence results for the cell-vertex finite volume scheme $(2.9)-(2.12)$. While the analysis is rather intricate, it ultimately shows that the scheme yields accurate results on certain subsets of $\Omega$ where $u$ is smooth; see Remark 4.2 below for more details. 
We first derive various interpolation errors. Set

$$
I=\{(i, j): i=1, \ldots, N-1, j=1, \ldots, M\} .
$$

We have

Lemma 4.1. For any $(i, j) \in I$, assume that $u \in C^{3}\left(K_{i, j} \cup K_{i+1, j}\right)$. Then

$$
\begin{aligned}
&\left|\mu \eta_{i, j}\right|+\left|\mu\left(a \eta_{x}+r \eta_{t}\right)_{i, j}\right| \leq C\left(h_{i} k_{j}\right)^{-1 / 2} h^{2}|u|_{H^{3}\left(K_{i, j}\right)}, \\
&\left|\mu_{x} \eta_{i, 0}\right| \leq C h_{i}^{3 / 2}\left|u^{0}\right|_{H^{3}\left(x_{i-1}, x_{i}\right)}, \\
&\left|\mu_{t}\left(u_{x}\right)_{i, j}-\hat{\mu}_{t}\left(u_{x}^{I}\right)_{i, j}\right| \leq C\left\{\left|h_{i}-h_{i+1}\right|\|u\|_{C^{2}\left(\bar{K}_{i, j}\right)}\right. \\
&\left.+\left(h_{i+1}^{2}+h_{i}^{2}+k_{j}^{2}\right)\|u\|_{C^{3}\left(K_{i, j} \cup K_{i+1, j}\right)}\right\}
\end{aligned}
$$

with

$$
\begin{aligned}
\left|\mu_{t}\left(u_{x}\right)_{0, j}-\hat{\mu}_{t}\left(u_{x}^{I}\right)_{0, j}\right| \leq & C\left\{\left|h_{1}-h_{2}\right|\|u\|_{C^{2}\left(\bar{K}_{1, j}\right)}\right. \\
& \left.+\left(h_{2}^{2}+h_{1}^{2}+k_{j}^{2}\right)\|u\|_{C^{3}\left(K_{1, j} \cup K_{2, j}\right)}\right\},
\end{aligned}
$$

where $|\cdot|_{H^{3}(D)}$ denotes the usual seminorm on $H^{3}(D)$.

Proof. The proof of (4.1) can be found in the proof of Theorem 4 in Morton and Süli [10]. In a similar manner one can prove (4.2).

We need only prove (4.3) and (4.4). For $i \geq 1$, using (2.12), we have

$$
\begin{aligned}
\hat{\mu}_{t}\left(u_{x}^{I}\right)_{i, j}= & \frac{2}{h_{i}+h_{i+1}}\left(\mu u_{i+1, j}^{I}-\mu u_{i, j}^{I}\right) \\
= & \frac{1}{2\left(h_{i}+h_{i+1}\right)}\left(u_{i+1, j}+u_{i+1, j-1}-u_{i-1, j}-u_{i-1, j-1}\right), \quad \text { by }(2.4), \\
= & \frac{1}{2}\left(u_{x}\left(x_{i}, t_{j}\right)+u_{x}\left(x_{i}, t_{j-1}\right)\right)+\frac{h_{i+1}-h_{i}}{4}\left(u_{x x}\left(x_{i}, t_{j}\right)+u_{x x}\left(x_{i}, t_{j-1}\right)\right) \\
& +\frac{1}{12\left(h_{i}+h_{i+1}\right)}\left\{h_{i+1}^{3}\left(u_{x x x}\left(\theta_{1}, t_{j}\right)+u_{x x x}\left(\theta_{2}, t_{j-1}\right)\right)\right. \\
& \left.\quad+h_{i}^{3}\left(u_{x x x}\left(\theta_{3}, t_{j}\right)+u_{x x x}\left(\theta_{4}, t_{j-1}\right)\right)\right\}
\end{aligned}
$$

by a Taylor expansion, where

$$
x_{i-1}<\theta_{3}, \theta_{4}<x_{i}<\theta_{1}, \theta_{2}<x_{i+1} .
$$

Thus,

$$
\begin{aligned}
\mid \mu_{t}\left(u_{x}\right)_{i, j}- & \hat{\mu}_{t}\left(u_{x}^{I}\right)_{i, j} \mid \\
\leq & \left|\frac{1}{k_{j}} \int_{t_{j-1}}^{t_{j}} u_{x}\left(x_{i}, t\right) d t-\frac{1}{2}\left(u_{x}\left(x_{i}, t_{j}\right)+u_{x}\left(x_{i}, t_{j-1}\right)\right)\right| \\
& \quad+C\left|h_{i+1}-h_{i}\right|\|u\|_{C^{2}\left(\bar{K}_{i, j}\right)}+C\left(h_{i+1}^{2}+h_{i}^{2}\right)\|u\|_{C^{3}\left(K_{i, j} \cup K_{i+1, j}\right)} \\
\leq & C\left|h_{i+1}-h_{i}\right|\|u\|_{C^{2}\left(\bar{K}_{i, j}\right)}+C\left(h_{i+1}^{2}+h_{i}^{2}+k_{j}^{2}\right)\|u\|_{C^{3}\left(K_{i, j} \cup K_{i+1, j}\right)},
\end{aligned}
$$

by the error estimate for the trapezoidal rule. 
Similarly, for $i=0$, by a Taylor expansion about $x=0$ and using $u_{0, j}=0$ for all $j$, we have

$$
\begin{aligned}
\hat{\mu}_{t}\left(u_{x}^{I}\right)_{0, j}= & \frac{2}{h_{1}} \mu_{t} u_{1, j}^{I}-\mu_{t}\left(u_{x}^{I}\right)_{1, j} \\
= & \frac{1}{h_{1}}\left(u_{1, j}+u_{1, j-1}\right)-\frac{1}{2\left(h_{1}+h_{2}\right)}\left(u_{2, j}+u_{2, j-1}\right) \\
= & u_{x}\left(0, t_{j}\right)+u_{x}\left(0, t_{j-1}\right)+\frac{h_{1}}{2}\left(u_{x x}\left(0, t_{j}\right)+u_{x x}\left(0, t_{j-1}\right)\right) \\
& +\frac{h_{1}^{2}}{6}\left(u_{x x x}\left(\theta_{5}, t_{j}\right)+u_{x x x}\left(\theta_{6}, t_{j-1}\right)\right) \\
& -\frac{1}{2}\left(u_{x}\left(0, t_{j}\right)+u_{x}\left(0, t_{j-1}\right)\right)-\frac{h_{1}+h_{2}}{4}\left(u_{x x}\left(0, t_{j}\right)+u_{x x}\left(0, t_{j-1}\right)\right) \\
& -\frac{\left(h_{1}+h_{2}\right)^{2}}{12}\left(u_{x x x}\left(\theta_{7}, t_{j}\right)+u_{x x x}\left(\theta_{8}, t_{j-1}\right)\right) \\
= & \frac{1}{2}\left(u_{x}\left(0, t_{j}\right)+u_{x}\left(0, t_{j-1}\right)\right)+\frac{h_{1}-h_{2}}{4}\left(u_{x x}\left(0, t_{j}\right)+u_{x x}\left(0, t_{j-1}\right)\right) \\
& +\frac{h_{1}^{2}}{6}\left(u_{x x x}\left(\theta_{5}, t_{j}\right)+u_{x x x}\left(\theta_{6}, t_{j-1}\right)\right) \\
& -\frac{\left(h_{1}+h_{2}\right)^{2}}{12}\left(u_{x x x}\left(\theta_{7}, t_{j}\right)+u_{x x x}\left(\theta_{8}, t_{j-1}\right)\right)
\end{aligned}
$$

where

$$
0<\theta_{l}<x_{2} \quad \text { for } l=5, \ldots, 8
$$

Hence,

$$
\begin{aligned}
\mid \mu_{t}\left(u_{x}\right)_{0, j}- & \hat{\mu}_{t}\left(u_{x}^{I}\right)_{0, j} \mid \\
\leq & \left|\frac{1}{k_{j}} \int_{t_{j-1}}^{t_{j}} u_{x}(0, t) d t-\frac{1}{2}\left(u_{x}\left(0, t_{j}\right)+u_{x}\left(0, t_{j-1}\right)\right)\right| \\
& +C\left|h_{1}-h_{2}\right|\|u\|_{C^{2}\left(\bar{K}_{1, j}\right)}+C\left(h_{2}^{2}+h_{1}^{2}\right)\|u\|_{C^{3}\left(K_{1, j} \cup K_{2, j}\right)} \\
\leq & C\left|h_{1}-h_{2}\right|\|u\|_{C^{2}\left(\bar{K}_{1, j}\right)}+C\left(h_{2}^{2}+h_{1}^{2}+k_{j}^{2}\right)\|u\|_{C^{3}\left(K_{1, j} \cup K_{2, j}\right)},
\end{aligned}
$$

which completes the proof of (4.4).

For each $(i, j) \in I$, define

$$
B_{i, j}(w)=-\frac{\varepsilon}{h_{i}}\left(\hat{\mu}_{t}\left(w_{x}\right)_{i, j}-\hat{\mu}_{t}\left(w_{x}\right)_{i-1, j}\right)+\mu\left(a w_{x}+r w_{t}+b w\right)_{i, j},
$$

for all $w$ for which the right-hand side is defined. Then

$$
\hat{B}(w, p)=\sum_{j=1}^{M} \sum_{i=1}^{N-1} h_{i} k_{j} \mu p_{i, j} B_{i, j}(w) .
$$

For $\tilde{I}$ any nonempty subset of $I$, let $\tilde{\Omega}=\bigcup_{(i, j) \in \tilde{I}} K_{i, j}$. Set

$$
|w|_{\hat{B}(\tilde{\Omega})}=\left\{\sum_{(i, j) \in \tilde{I}} h_{i} k_{j}\left|B_{i, j}(w)\right|^{2}\right\}^{1 / 2} .
$$


We note that $|\cdot|_{\hat{B}\left(\Omega^{h}\right)}$ is a seminorm on $L^{2}(\Omega)$. It can be regarded as a generalization of the seminorm $\left|\nabla \cdot I^{h}(a(\cdot))\right|_{l_{2}\left(\Omega^{h}\right)}$ introduced in Morton and Stynes [9].

Using Lemma 4.1, we get the following error bound in a local $|\cdot|_{\hat{B}\left(\Omega^{h}\right)}$ seminorm.

Theorem 4.1. Let $\tilde{\Omega}=\bigcup_{(i, j) \in \tilde{I}} K_{i, j}$ be arbitrary. Set

$$
\tilde{I}^{+}=\left\{(i, j) \in I: j=j^{\prime} \text { and }\left|i-i^{\prime}\right| \leq 1 \quad \text { for some }\left(i^{\prime}, j^{\prime}\right) \in \tilde{I}\right\} .
$$

Let $\tilde{\Omega}^{+}=\bigcup_{(i, j) \in \tilde{I}^{+}} K_{i, j}$. Assume that $u \in C^{3}\left(\tilde{\Omega}^{+}\right)$. Then

$$
\begin{aligned}
\mid u^{h}- & \left.u^{I}\right|_{\hat{B}(\tilde{\Omega})} \\
\leq & C \varepsilon\left\{\max _{(i, j) \in \tilde{I}}\left\{h_{i}^{-1}\left|h_{i+1}-h_{i}\right|, h_{i}^{-1}\left|h_{i}-h_{i-1}\right|\right\}\|u\|_{C^{2}\left(\tilde{\Omega}^{+}\right)}\right. \\
& \left.+\max _{(i, j) \in \tilde{I}}\left\{h_{i+1}^{2} h_{i}^{-1}, h_{i}, k_{j}^{2} h_{i}^{-1}\right\}\|u\|_{C^{3}\left(\tilde{\Omega}^{+}\right)}\right\}+C h^{2}|u|_{H^{3}(\tilde{\Omega})} .
\end{aligned}
$$

Proof. From (1.1), (2.9) and (2.11), we get

$$
\begin{aligned}
\hat{B}\left(u^{h}-u^{I}, p\right)= & -\varepsilon \sum_{j=1}^{M} \sum_{i=1}^{N-1} k_{j} \mu p_{i, j}\left\{e_{i, j}-e_{i-1, j}\right\} \\
& +\left(a \eta_{x}+r \eta_{t}+b \eta, p\right), \quad \forall p \in \mathcal{M}^{h},
\end{aligned}
$$

where $e_{i, j}$ is as in (3.11).

Fix $(i, j) \in \tilde{I}$. Take $p$ in (4.9) to be the characteristic function of $K_{i, j}$. From (4.6) this yields

$$
B_{i, j}\left(u^{h}-u^{I}\right)=-\varepsilon h_{i}^{-1}\left\{e_{i, j}-e_{i-1, j}\right\}+\mu\left(a \eta_{x}+r \eta_{t}+b \eta\right)_{i, j} .
$$

Applying Lemma 4.1 gives

$$
\begin{aligned}
\left|B_{i, j}\left(u^{h}-u^{I}\right)\right| \leq & C \varepsilon h_{i}^{-1}\left\{\left(\left|h_{i+1}-h_{i}\right|+\left|h_{i}-h_{i-1}\right|\right)\|u\|_{C^{2}\left(\bar{K}_{i-1, j} \cup \bar{K}_{i, j}\right)}\right. \\
& \left.+\left(h_{i+1}^{2}+h_{i}^{2}+h_{i-1}^{2}+k_{j}^{2}\right)\|u\|_{C^{3}\left(K_{i-1, j} \cup K_{i, j} \cup K_{i+1, j}\right)}\right\} \\
& +C\left(h_{i} k_{j}\right)^{-1 / 2} h^{2}|u|_{H^{3}\left(K_{i, j}\right)},
\end{aligned}
$$

with the convention that $h_{0}=h_{1}$. The desired inequality follows immediately from the definition (4.7).

In what follows, we shall derive a local error bound in an energy seminorm. To this end, we introduce a cutoff function $w(x, t)$ defined by

$$
\omega(x, t)=g\left(\frac{x-x^{*}}{\gamma h}\right) g\left(t-t^{*}\right),
$$

where $\left(x^{*}, t^{*}\right)$ is a fixed node, $\gamma \geq 1$ is a constant (which we choose later to be independent of $\varepsilon$ and the mesh), and

$$
g(r)=\frac{2}{1+\exp (r)} \quad \forall r \in(-\infty, \infty) .
$$


Set

$$
\begin{aligned}
& \Omega_{0}=\left\{(x, t) \in \Omega: x \leq x^{*}, t \leq t^{*}\right\}, \\
& \Omega_{0}^{+}=\left\{(x, t) \in \Omega: x \leq x^{*}+s \gamma h \ln \frac{1}{h}, t \leq t^{*}+s \gamma h \ln \frac{1}{h}\right\},
\end{aligned}
$$

where $s>0$ is some integer (which we choose later to be independent of $\varepsilon$ and $h$ ). Without loss of generality, we assume that $\Omega_{0}^{+}$consists of cells, that is,

$$
\Omega_{0}^{+}=\bigcup_{j=1}^{j^{\prime}} \bigcup_{i=1}^{i^{\prime}} K_{i, j}
$$

for some $\left(i^{\prime}, j^{\prime}\right) \in I$. Set

$$
\Omega_{0}^{++}=\bigcup_{j=1}^{j^{\prime}} \bigcup_{i=1}^{i^{\prime}+1} K_{i, j}
$$

One can easily show that

$$
\begin{gathered}
\omega_{x}<0 \quad \text { and } \quad \omega_{t}<0 \quad \text { on } \Omega, \\
\max _{D_{i, j}} \omega / \min _{D_{i, j}} \omega \leq C, \quad \max _{D_{i, j}}\left|\omega_{x}\right| / \min _{D_{i, j}}\left|\omega_{x}\right| \leq C,
\end{gathered}
$$

where $D_{i, j}=\overline{K_{i, j} \cup K_{i+1, j}}$,

$$
\begin{gathered}
\left|\omega_{x}\right| \leq C \gamma^{-1} h^{-1} \omega, \\
\omega\left(x_{i^{\prime}}, t\right) \leq C h^{s} \quad \text { for } t \in[0, T], \\
\text { and } \omega(x, t) \geq 1 \quad \text { on } \Omega_{0} .
\end{gathered}
$$

Notation. We introduce the following weighted norms:

$$
\begin{aligned}
&|v|_{l_{2}\left(\Omega_{0}^{+}\right), \omega}=\left\{\sum_{j=1}^{j^{\prime}} \sum_{i=1}^{i^{\prime}} h_{i} k_{j} \omega_{i, j}\left|\mu v_{i, j}\right|^{2}\right\}^{1 / 2}, \\
&|v|_{l_{2}\left(\partial_{-} \Omega_{0}^{+}\right), \omega}=\left\{\sum_{i=1}^{i^{\prime}} h_{i} \omega_{i, 1}\left|\mu_{x} v_{i, 0}\right|^{2}\right\}^{1 / 2}, \\
&\left|v_{x}\right|_{\hat{l}_{2}\left(\Omega_{0}^{+}\right), \omega}=\left\{\sum_{j=1}^{j^{\prime}} \sum_{i=1}^{i^{\prime}} \frac{h_{i}+h_{i+1}}{2} k_{j} \omega_{i, j}\left|\hat{\mu}_{t}\left(v_{x}\right)_{i, j}\right|^{2}\right. \\
&\left.+\frac{h_{1}}{4} \sum_{j=1}^{j^{\prime}} k_{j} \omega_{1, j}\left|\hat{\mu}_{t}\left(v_{x}\right)_{0, j}\right|^{2}\right\}^{1 / 2},
\end{aligned}
$$

for all $v(x, t)$ for which the right-hand sides are defined.

Define $R_{\omega}: \mathcal{U}_{0}^{h} \rightarrow \mathcal{M}^{h}$ by

$$
R_{\omega} v= \begin{cases}\omega_{i, j} \mu v_{i, j} & \text { on } K_{i, j}, \text { for } i=1, \ldots, i^{\prime}, j=1, \ldots, j^{\prime}, \\ 0 & \text { otherwise. }\end{cases}
$$

Then we can prove a weighted Gårding inequality. 
Lemma 4.2. Assume that there exists a positive constant $c_{0}$, which is independent of $\varepsilon$ and of the mesh, such that

$$
\varepsilon \leq c_{0} h_{i} \quad \text { for } i=1, \ldots, i^{\prime}+1 .
$$

Then

$$
\begin{aligned}
\hat{B}\left(v, R_{\omega} v\right) \geq & \frac{\varepsilon}{4}\left|v_{x}\right|_{\hat{l}_{2}\left(\Omega_{0}^{+}\right), \omega}+b|v|_{l_{2}\left(\Omega_{0}^{+}\right), \omega}^{2} \\
& -\frac{r}{2}|v|_{l_{2}\left(\partial_{-} \Omega_{0}^{+}\right), \omega}^{2}-C h^{s} h_{i^{\prime}+1}^{-1}|v|_{l_{2}\left(\Omega_{0}^{++}\right)}^{2},
\end{aligned}
$$

where $|\cdot|_{l_{2}\left(\Omega_{0}^{++}\right)}$is defined analogously to $|\cdot|_{l_{2}\left(\Omega^{h}\right)}$.

Proof. Similarly to the derivation of (3.7), we have

$$
\begin{aligned}
\left(a v_{x}+\right. & \left.r v_{t}+b v, R_{\omega} v\right) \\
= & \frac{a}{2} \sum_{j=1}^{j^{\prime}} \sum_{i=1}^{i^{\prime}} k_{j} \omega_{i, j}\left(\left|\mu_{t} v_{i, j}\right|^{2}-\left|\mu_{t} v_{i-1, j}\right|^{2}\right) \\
& +\frac{r}{2} \sum_{j=1}^{j^{\prime}} \sum_{i=1}^{i^{\prime}} h_{i} \omega_{i, j}\left(\left|\mu_{x} v_{i, j}\right|^{2}-\left|\mu_{x} v_{i, j-1}\right|^{2}\right) \\
& +b \sum_{j=1}^{j^{\prime}} \sum_{i=1}^{i^{\prime}} h_{i} k_{j} \omega_{i, j}\left|\mu v_{i, j}\right|^{2} \\
= & \frac{a}{2} \sum_{j=1}^{j^{\prime}} k_{j}\left\{\sum_{i=1}^{i^{\prime}}\left(\omega_{i, j}-\omega_{i+1, j}\right)\left|\mu_{t} v_{i, j}\right|^{2}+\omega_{i^{\prime}+1, j}\left|\mu_{t} v_{i^{\prime}, j}\right|^{2}\right\} \\
& +\frac{r}{2} \sum_{i=1}^{i^{\prime}} h_{i}\left\{\sum_{j=1}^{j^{\prime}}\left(\omega_{i, j}-\omega_{i, j+1}\right)\left|\mu_{x} v_{i, j}\right|^{2}+\omega_{i, j^{\prime}+1}\left|\mu_{x} v_{i, j^{\prime}}\right|^{2}-\omega_{i, 1}\left|\mu_{x} v_{i, 0}\right|^{2}\right\} \\
& +b \sum_{j=1}^{j^{\prime}} \sum_{i=1}^{i^{\prime}} h_{i} k_{j} \omega_{i, j}\left|\mu v_{i, j}\right|^{2},
\end{aligned}
$$

by summation by parts and using $\mu_{t} v_{0, j}=0$.

Next, the contribution to $\hat{B}\left(v, R_{\omega} v\right)$ from the diffusion term is

$$
\begin{array}{r}
Q_{\varepsilon} \equiv-\varepsilon \sum_{j=1}^{j^{\prime}} \sum_{i=1}^{i^{\prime}} k_{j} \omega_{i, j} \mu v_{i, j}\left\{\hat{\mu}_{t}\left(v_{x}\right)_{i, j}-\hat{\mu}_{t}\left(v_{x}\right)_{i-1, j}\right\} \\
=\varepsilon \sum_{j=1}^{j^{\prime}} k_{j}\left\{-\omega_{i^{\prime}, j} \mu v_{i^{\prime}, j} \hat{\mu}_{t}\left(v_{x}\right)_{i^{\prime}, j}+\omega_{1, j} \mu v_{1, j} \hat{\mu}_{t}\left(v_{x}\right)_{0, j}\right. \\
\left.-\sum_{i=1}^{i^{\prime}-1}\left(\omega_{i, j} \mu v_{i, j}-\omega_{i+1, j} \mu v_{i+1, j}\right) \hat{\mu}_{t}\left(v_{x}\right)_{i, j}\right\} .
\end{array}
$$


Now, analogously to (3.3), we have

$$
\begin{aligned}
-\mu v_{i^{\prime}, j} & \hat{\mu}_{t}\left(v_{x}\right)_{i^{\prime}, j} \\
& \geq \frac{h_{i^{\prime}}+h_{i^{\prime}+1}}{4}\left|\hat{\mu}_{t}\left(v_{x}\right)_{i^{\prime}, j}\right|^{2}-\frac{1}{h_{i^{\prime}}+h_{i^{\prime}+1}}\left|\mu v_{i^{\prime}+1, j}\right|^{2} .
\end{aligned}
$$

For $i=1, \ldots, i^{\prime}-1$,

$$
\begin{aligned}
& -\left(\omega_{i, j} \mu v_{i, j}-\omega_{i+1, j} \mu v_{i+1, j}\right) \hat{\mu}_{t}\left(v_{x}\right)_{i, j} \\
& \quad=\left\{\omega_{i, j}\left(\mu v_{i+1, j}-\mu v_{i, j}\right)-\left(\omega_{i, j}-\omega_{i+1, j}\right) \mu v_{i+1, j}\right\} \hat{\mu}_{t}\left(v_{x}\right)_{i, j} \\
& \quad=\frac{h_{i}+h_{i+1}}{2} \omega_{i, j}\left|\hat{\mu}_{t}\left(v_{x}\right)_{i, j}\right|^{2}-\left(\omega_{i, j}-\omega_{i+1, j}\right) \mu v_{i+1, j} \hat{\mu}_{t}\left(v_{x}\right)_{i, j}, \quad \text { by }(2.12), \\
& \quad \geq \frac{h_{i}+h_{i+1}}{4} \omega_{i, j}\left|\hat{\mu}_{t}\left(v_{x}\right)_{i, j}\right|^{2}-\frac{1}{h_{i}+h_{i+1}}\left(\omega_{i, j}-\omega_{i+1, j}\right)^{2} \omega_{i, j}^{-1}\left|\mu v_{i+1, j}\right|^{2} .
\end{aligned}
$$

Substituting (4.22), (3.4) and (4.23) into (4.21), we obtain

$$
\begin{aligned}
Q_{\varepsilon} \geq & \varepsilon \sum_{j=1}^{j^{\prime}} k_{j}\left\{\frac{h_{i^{\prime}}+h_{i^{\prime}+1}}{4} \omega_{i^{\prime}, j}\left|\hat{\mu}_{t}\left(v_{x}\right)_{i^{\prime}, j}\right|^{2}-\frac{1}{h_{i^{\prime}}+h_{i^{\prime}+1}} \omega_{i^{\prime}, j}\left|\mu v_{i^{\prime}+1, j}\right|^{2}\right. \\
& +\frac{h_{1}}{8} \omega_{1, j}\left(\left|\hat{\mu}_{t}\left(v_{x}\right)_{0, j}\right|^{2}-\left|\hat{\mu}_{t}\left(v_{x}\right)_{1, j}\right|^{2}\right)+\sum_{i=1}^{i^{\prime}-1} \frac{h_{i}+h_{i+1}}{4} \omega_{i, j}\left|\hat{\mu}_{t}\left(v_{x}\right)_{i, j}\right|^{2} \\
& \left.-\sum_{i=1}^{i^{\prime}-1} \frac{1}{h_{i}+h_{i+1}}\left(\omega_{i, j}-\omega_{i+1, j}\right)^{2} \omega_{i, j}^{-1}\left|\mu v_{i+1, j}\right|^{2}\right\} \\
\geq & \frac{\varepsilon}{4}\left|v_{x}\right|_{\hat{l}_{2}\left(\Omega_{0}^{+}\right), \omega}^{2} \frac{\varepsilon}{h_{i^{\prime}}+h_{i^{\prime}+1}} \sum_{j=1}^{j^{\prime}} k_{j} \omega_{i^{\prime}, j}\left|\mu v_{i^{\prime}+1, j}\right|^{2} \\
& -\varepsilon \sum_{j=1}^{j^{\prime}} k_{j} \sum_{i=1}^{i^{\prime}-1} \frac{1}{h_{i}+h_{i+1}}\left(\omega_{i, j}-\omega_{i+1, j}\right)^{2} \omega_{i, j}^{-1}\left|\mu v_{i+1, j}\right|^{2} .
\end{aligned}
$$

In (4.24), we can bound the second term by using (4.17), to get

$$
\begin{aligned}
& \frac{\varepsilon}{h_{i^{\prime}}+h_{i^{\prime}+1}} \sum_{j=1}^{j^{\prime}} k_{j} \omega_{i^{\prime}, j}\left|\mu v_{i^{\prime}+1, j}\right|^{2} \\
& \quad \leq C \frac{\varepsilon}{h_{i^{\prime}}+h_{i^{\prime}+1}} h^{s} \sum_{j=1}^{j^{\prime}} k_{j}\left|\mu v_{i^{\prime}+1, j}\right|^{2} \\
& \leq C h^{s} h_{i^{\prime}+1}^{-1}|v|_{l_{2}\left(\Omega_{0}^{++}\right)}^{2},
\end{aligned}
$$

using (4.19). 
As for the last term in (4.24), using (2.6) and $(a+b)^{2} \leq 2\left(a^{2}+b^{2}\right)$, we get $(4.26)$

$$
\begin{aligned}
& \sum_{i=1}^{i^{\prime}-1} \frac{1}{h_{i}+h_{i+1}}\left(\omega_{i, j}-\omega_{i+1, j}\right)^{2} \omega_{i, j}^{-1}\left|\mu v_{i+1, j}\right|^{2} \\
& \leq \frac{1}{2} \sum_{i=1}^{i^{\prime}-1} \frac{1}{h_{i}+h_{i+1}} h_{i+1}^{2} \max _{K_{i+1, j}}\left|\omega_{x}\right|^{2} \omega_{i, j}^{-1}\left(\left|\mu_{t} v_{i, j}\right|^{2}+\left|\mu_{t} v_{i+1, j}\right|^{2}\right) \\
& \leq C \sum_{i=1}^{i^{\prime}-1} \frac{h_{i+1}^{2}}{h_{i}+h_{i+1}} \gamma^{-1} h^{-1} \max _{K_{i+1, j}}\left|\omega_{x}\right|\left(\left|\mu_{t} v_{i, j}\right|^{2}+\left|\mu_{t} v_{i+1, j}\right|^{2}\right), \\
& \leq C \gamma^{-1} \sum_{i=1}^{i^{\prime}-1} \max _{K_{i+1, j}}\left|\omega_{x}\right|\left(\left|\mu_{t} v_{i, j}\right|^{2}+\left|\mu_{t} v_{i+1, j}\right|^{2}\right) \\
& \leq C \gamma^{-1} \sum_{i=1}^{i^{\prime}}\left(\max _{K_{i+1, j}}\left|\omega_{x}\right|+\max _{K_{i, j}}\left|\omega_{x}\right|\right)\left|\mu_{t} v_{i, j}\right|^{2} \\
& \leq C \gamma^{-1} \sum_{i=1}^{i^{\prime}}\left(\max _{K_{i, j} \cup K_{i+1, j}}\left|\omega_{x}\right| / \min _{K_{i+1, j}}\left|\omega_{x}\right|\right) \frac{1}{h_{i+1}}\left(\omega_{i, j}-\omega_{i+1, j}\right)\left|\mu_{t} v_{i, j}\right|^{2}, \\
& \quad \leq C \gamma^{-1} \sum_{i=1}^{i^{\prime}} \frac{1}{h_{i+1}}\left(\omega_{i, j}-\omega_{i+1, j}\right)\left|\mu_{t} v_{i, j}\right|^{2}, \quad \text { and }(4.15), \\
& \leq \frac{a}{2 c_{0}} \sum_{i=1}^{i^{\prime}} \frac{1}{h_{i+1}}\left(\omega_{i, j}-\omega_{i+1, j}\right)\left|\mu_{t} v_{i, j}\right|^{2},
\end{aligned}
$$

on choosing $\gamma$ sufficiently large, independently of $\varepsilon$ and of the mesh used.

Thus, from (4.24) - (4.26) and (4.19), we obtain

$$
Q_{\varepsilon} \geq \frac{\varepsilon}{4}\left|v_{x}\right|_{\hat{l}_{2}\left(\Omega_{0}^{+}\right), \omega}^{2}-\frac{a}{2} \sum_{j=1}^{j^{\prime}} k_{j} \sum_{i=1}^{i^{\prime}}\left(\omega_{i, j}-\omega_{i+1, j}\right)\left|\mu_{t} v_{i, j}\right|^{2}-C h^{s} h_{i^{\prime}+1}^{-1}|v|_{l_{2}\left(\Omega_{0}^{++}\right)}^{2} .
$$

Combine this with (4.20) and use (4.14) to complete the proof.

We now prove the main result of this section.

Theorem 4.2. Assume that $\varepsilon \leq a\left(h_{N-1}+h_{N}\right)$ and that (4.19) holds. If $u \in$ $C^{3}\left(\Omega_{0}^{++}\right)$, then

$$
\begin{aligned}
\left|u-u^{h}\right|_{E\left(\Omega_{0}\right)} \leq & C h\left(\|u\|_{C^{3}\left(\Omega_{0}^{++}\right)}+\left|u^{0}\right|_{H^{3}\left(0, x_{i^{\prime}}\right)}\right) \\
& +C\left(h^{s} h_{i^{\prime}+1}^{-1}\right)^{1 / 2}\left(\|f\|_{L^{2}(\Omega)}+\left\|u^{0}\right\|_{L^{2}(0,1)}+|u|_{H^{3}\left(\Omega_{0}^{++}\right)}\right),
\end{aligned}
$$

where $\Omega_{0}$ and $\Omega_{0}^{++}$are as in (4.11) and (4.13) respectively, $s$ is as in (4.12), and

$$
\left|u-u^{h}\right|_{E\left(\Omega_{0}\right)}=\left\{\varepsilon\left|\mu_{t}\left(u_{x}\right)-\hat{\mu}_{t}\left(u_{x}^{h}\right)\right|_{l_{2}^{\circ}\left(\Omega_{0}\right)}^{2}+\left|u-u^{h}\right|_{l_{2}\left(\Omega_{0}\right)}^{2}\right\}^{1 / 2}
$$

with $\left|\mu_{t}\left(u_{x}\right)-\hat{\mu}_{t}\left(u_{x}^{h}\right)\right|_{l_{2}^{\circ}\left(\Omega_{0}\right)}$ and $|\cdot| l_{l_{2}\left(\Omega_{0}\right)}$ defined similarly to (3.9) and $|\cdot| l_{l_{2}\left(\Omega^{h}\right)}$. 
Proof. Applying Lemma 4.2, we obtain

$$
\begin{aligned}
& \frac{\varepsilon}{4}\left|\xi_{x}\right|_{\hat{l}_{2}\left(\Omega_{0}^{+}\right), \omega}^{2}+b|\xi|_{l_{2}\left(\Omega_{0}^{+}\right), \omega}^{2} \\
& \quad \leq \hat{B}\left(\xi, R_{\omega} \xi\right)+\frac{r}{2}|\xi|_{l_{2}\left(\partial_{-} \Omega_{0}^{+}\right), \omega}^{2}+C h^{s} h_{i^{\prime}+1}^{-1}|\xi|_{l_{2}\left(\Omega_{0}^{++}\right)}^{2}
\end{aligned}
$$

Recalling (4.6), we obtain

$$
\begin{aligned}
\hat{B}\left(\xi, R_{\omega} \xi\right) & =\sum_{j=1}^{j^{\prime}} \sum_{i=1}^{i^{\prime}} h_{i} k_{j} \omega_{i, j} \mu \xi_{i, j} B_{i, j}(\xi) \\
& \leq \frac{b}{2}|\xi|_{l_{2}\left(\Omega_{0}^{+}\right), \omega}^{2}+\frac{1}{2 b} \sum_{j=1}^{j^{\prime}} \sum_{i=1}^{i^{\prime}} h_{i} k_{j} \omega_{i, j}\left|B_{i, j}(\xi)\right|^{2} .
\end{aligned}
$$

Put this into (4.27) to get

$$
\begin{aligned}
& \frac{\varepsilon}{2}\left|\xi_{x}\right|_{\hat{l}_{2}\left(\Omega_{0}^{+}\right), \omega+b|\xi|_{l_{2}\left(\Omega_{0}^{+}\right), \omega}^{2}} \\
& \quad \leq \frac{1}{b} \sum_{j=1}^{j^{\prime}} \sum_{i=1}^{i^{\prime}} h_{i} k_{j} \omega_{i, j}\left|B_{i, j}(\xi)\right|^{2}+r|\xi|_{l_{2}\left(\partial_{-} \Omega_{0}^{+}\right), \omega}^{2}+C h^{s} h_{i^{\prime}+1}^{-1}|\xi|_{l_{2}\left(\Omega_{0}^{++}\right)}^{2} \\
& \quad \leq C\left\{|\xi|_{\hat{B}\left(\Omega_{0}^{+}\right)}^{2}+|\xi|_{l_{2}\left(\partial_{-} \Omega_{0}^{+}\right)}^{2}\right\}+C h^{s} h_{i^{\prime}+1}^{-1}|\xi|_{l_{2}\left(\Omega_{0}^{++}\right)}^{2},
\end{aligned}
$$

since $\omega(x, t) \leq 2$ on $\bar{\Omega}$, where $|\cdot|_{l_{2}\left(\partial_{-} \Omega_{0}^{+}\right)}$is defined similarly to $|\cdot|_{l_{2}\left(\partial_{-} \Omega^{h}\right)}$.

Appealing to Theorem 4.1, we obtain

$$
\begin{aligned}
|\xi|_{\hat{B}\left(\Omega_{0}^{+}\right)} \leq & C \varepsilon \max _{1 \leq i \leq i^{\prime}}\left\{h_{i}^{-1}\right\}\left\{\max _{1 \leq i \leq i^{\prime}}\left\{\left|h_{i+1}-h_{i}\right|\right\}\|u\|_{C^{2}\left(\Omega_{0}^{+}\right)}+h^{2}\|u\|_{C^{3}\left(\Omega_{0}^{++}\right)}\right\} \\
& +C h^{2}|u|_{H^{3}\left(\Omega_{0}^{+}\right)} \\
\leq & C h\|u\|_{C^{3}\left(\Omega_{0}^{++}\right)}
\end{aligned}
$$

using (4.19).

By $(2.10)$,

$$
|\xi|_{l_{2}\left(\partial_{-} \Omega_{0}^{+}\right)}=|\eta|_{l_{2}\left(\partial_{-} \Omega_{0}^{+}\right)} \leq C h^{2}\left|u^{0}\right|_{H^{3}\left(0, x_{i^{\prime}}\right)},
$$

by virtue of (4.2).

For the last term in (4.29), we have

$$
\begin{aligned}
|\xi|_{l_{2}\left(\Omega_{0}^{++}\right)} & \leq\left|u^{h}\right|_{l_{2}\left(\Omega_{0}^{++}\right)}+|u|_{l_{2}\left(\Omega_{0}^{++}\right)}+|\eta|_{l_{2}\left(\Omega_{0}^{++}\right)} \\
& \leq C\left(|f|_{l_{2}\left(\Omega^{h}\right)}+\left|u^{0}\right|_{l_{2}\left(\partial_{-} \Omega^{h}\right)}+|u|_{l_{2}\left(\Omega_{0}^{++}\right)}\right)+C h^{2}|u|_{H^{3}\left(\Omega_{0}^{++}\right)},
\end{aligned}
$$

according to Theorem 3.2 and (4.1).

Clearly, for any $w \in L^{2}(\Omega)$,

$$
|w|_{l_{2}\left(\Omega_{0}^{++}\right)} \leq|w|_{l_{2}\left(\Omega^{h}\right)} \leq\|w\|_{L_{2}(\Omega)},
$$

by a Cauchy-Schwarz inequality. Also,

$$
\|u\|_{L^{2}(\Omega)} \leq C\left(\|f\|_{L^{2}(\Omega)}+\left\|u^{0}\right\|_{L^{2}(0,1)}\right) .
$$


Thus,

$$
|\xi|_{l_{2}\left(\Omega_{0}^{++}\right)} \leq C\left(\|f\|_{L^{2}(\Omega)}+\left\|u^{0}\right\|_{L^{2}(0,1)}\right)+C h^{2}|u|_{H^{3}\left(\Omega_{0}^{++}\right)} .
$$

Collecting (4.30) - (4.32) into (4.29) yields

$$
\begin{aligned}
& \left\{\varepsilon\left|\xi_{x}\right|_{\hat{l}_{2}\left(\Omega_{0}^{+}\right), \omega}^{2}+|\xi|_{l_{2}\left(\Omega_{0}^{+}\right), \omega}^{2}\right\}^{1 / 2} \\
& \quad \leq C h\|u\|_{C^{3}\left(\Omega_{0}^{++}\right)}+C h^{2}\left|u^{0}\right|_{H^{3}\left(0, x_{i^{\prime}}\right)} \\
& \quad+C\left(h^{s} h_{i^{\prime}+1}^{-1}\right)^{1 / 2}\left(\|f\|_{L^{2}(\Omega)}+\left\|u^{0}\right\|_{L^{2}(0,1)}+|u|_{H^{3}\left(\Omega_{0}^{++}\right)}\right) .
\end{aligned}
$$

Note that by (4.18),

$$
\varepsilon\left|\xi_{x}\right|_{\hat{l}_{2}\left(\Omega_{0}^{+}\right)}^{2}+|\xi|_{l_{2}\left(\Omega_{0}\right)}^{2} \leq \varepsilon\left|\xi_{x}\right|_{\hat{l}_{2}\left(\Omega_{0}^{+}\right), \omega}^{2}+|\xi|_{l_{2}\left(\Omega_{0}^{+}\right), \omega}^{2} .
$$

Combining (4.33) with (4.34), invoking the triangle inequality and using Lemma 4.1 , we obtain the desired result.

Remark 4.1. The assumption that $u \in C^{3}\left(\Omega_{0}^{++}\right)$in Theorem 4.2 can be guaranteed if the data is sufficiently smooth and satisfies certain compatibility conditions at the corner $(0,0)$ of $\Omega$; see Ladyženskaja et al. [7].

Corollary 4.1. Assume that the hypotheses of Theorem 4.2 hold and

$$
h_{i+1}=h_{i}+O\left(h^{2}\right) \quad \text { for } i=1, \ldots, i^{\prime}
$$

and

$$
h_{i^{\prime}+1}=O\left(h^{\kappa}\right) \quad \text { for some } \kappa>0 .
$$

Then

$$
\left|u-u^{h}\right|_{E\left(\Omega_{0}\right)} \leq C h^{2}\left(\|u\|_{C^{3}\left(\Omega_{0}^{++}\right)}+\left|u^{0}\right|_{H^{3}\left(0, x_{i^{\prime}}\right)}+\|f\|_{L^{2}(\Omega)}+\left\|u^{0}\right\|_{L^{2}(0,1)}\right) .
$$

Proof. By inspecting the proof of Theorem 4.2, we see (cf. (4.30)) that when (4.35) holds, then

$$
|\xi|_{\hat{B}\left(\Omega_{0}^{+}\right)} \leq C h^{2}\|u\|_{C^{3}\left(\Omega_{0}^{++}\right)} .
$$

Hence from (4.29), (4.37), (4.31) and (4.32), we have

$$
\begin{aligned}
& \left\{\varepsilon\left|\xi_{x}\right|_{\hat{l}_{2}\left(\Omega_{0}^{+}\right), \omega}^{2}+|\xi|_{l_{2}\left(\Omega_{0}^{+}\right), \omega}^{2}\right\}^{1 / 2} \\
& \leq C \\
& \quad C h^{2}\left(\|u\|_{C^{3}\left(\Omega_{0}^{++}\right)}+\left|u^{0}\right|_{H^{3}\left(0, x_{i^{\prime}}\right)}\right) \\
& \quad+C\left(h^{s} h_{i^{\prime}+1}^{-1}\right)^{1 / 2}\left(\|f\|_{L^{2}(\Omega)}+\left\|u^{0}\right\|_{L^{2}(0,1)}+|u|_{H^{3}\left(\Omega_{0}^{++}\right)}\right) .
\end{aligned}
$$

Since (4.36) implies $h_{i^{\prime}+1}^{-1}=O\left(h^{-\kappa}\right)$, we take $s=\kappa+4$ in (4.38). Now arguments exactly the same as in the proof of Theorem 4.2 lead to the desired result.

Corollary 4.2. Assume that the hypotheses of Theorem 4.2 hold and that there exists a positive constant $c_{1}$, which is independent of $\varepsilon$ and of the mesh, such that

$$
\varepsilon \leq c_{1} h_{i}^{2} \quad \text { for } i=1, \ldots, i^{\prime}+1 \text {. }
$$

Then

$$
\left|u-u^{h}\right|_{E\left(\Omega_{0}\right)} \leq C h^{2}\left(\|u\|_{C^{3}\left(\Omega_{0}^{++}\right)}+\left|u^{0}\right|_{H^{3}\left(0, x_{i^{\prime}}\right)}+\|f\|_{L^{2}(\Omega)}+\left\|u^{0}\right\|_{L^{2}(0,1)}\right) .
$$


Proof. From the proof of Lemma 4.2, we see that when (4.39) holds, one can get (cf. (4.25)), for each $v \in \mathcal{U}_{0}^{h}$,

$$
\hat{B}\left(v, R_{\omega} v\right) \geq \frac{\varepsilon}{4}\left|v_{x}\right|_{\hat{l}_{2}\left(\Omega_{0}^{+}\right), \omega}^{2}+b|v|_{l_{2}\left(\Omega_{0}^{+}\right), \omega}^{2}-\frac{r}{2}|v|_{l_{2}\left(\partial_{-} \Omega_{0}^{+}\right), \omega}^{2}-C h^{s}|v|_{l_{2}\left(\Omega_{0}^{++}\right)}^{2} .
$$

Hence, similarly to the derivation of (4.29), using (4.28), we obtain

$$
\begin{aligned}
& \frac{\varepsilon}{2}\left|\xi_{x}\right|_{\hat{l}_{2}\left(\Omega_{0}^{+}\right), \omega}^{2}+b|\xi|_{l_{2}\left(\Omega_{0}^{+}\right), \omega}^{2} \\
& \quad \leq \frac{1}{b} \sum_{j=1}^{j^{\prime}} \sum_{i=1}^{i^{\prime}} h_{i} k_{j} \omega_{i, j}\left|B_{i, j}(\xi)\right|^{2}+r|\xi|_{l_{2}\left(\partial_{-} \Omega_{0}^{+}\right), \omega}^{2}+C h^{s}|\xi|_{l_{2}\left(\Omega_{0}^{++}\right)}^{2} \\
& \quad \leq C\left\{|\xi|_{\hat{B}\left(\Omega_{0}^{+}\right)}^{2}+|\xi|_{l_{2}\left(\partial_{-} \Omega_{0}^{+}\right)}^{2}\right\}+C h^{s}|\xi|_{l_{2}\left(\Omega_{0}^{++}\right)}^{2} .
\end{aligned}
$$

Also, from (4.30) we see that (4.39) implies (4.37). It then follows from (4.40), (4.37), (4.31) and (4.32) that

$$
\begin{aligned}
& \left\{\varepsilon\left|\xi_{x}\right|_{\hat{l}_{2}\left(\Omega_{0}^{+}\right), \omega}^{2}+|\xi|_{l_{2}\left(\Omega_{0}^{+}\right), \omega}^{2}\right\}^{1 / 2} \\
& \leq \quad C h^{2}\left(\|u\|_{C^{3}\left(\Omega_{0}^{++}\right)}+\left|u^{0}\right|_{H^{3}\left(0, x_{i^{\prime}}\right)}\right) \\
& \quad+C h^{s / 2}\left(\|f\|_{L^{2}(\Omega)}+\left\|u^{0}\right\|_{L^{2}(0,1)}+|u|_{H^{3}\left(\Omega_{0}^{++}\right)}\right) .
\end{aligned}
$$

Choose $s=4$ and follow the same argument as in the proof of Theorem 4.2 to complete the proof.

Remark 4.2. The assumption (4.19) is reasonable, since we are interested in the singularly perturbed case. Theorem 4.2 tells us that under this assumption, away from any layers, the scheme $(2.9)-(2.12)$ on an arbitrary tensor product mesh is first-order accurate in the $l_{2}$ seminorm, as one can choose $s$ sufficiently large to make the term $C h^{s} h_{i^{\prime}+1}^{-1}$ negligible. Corollary 4.1 indicates that if we work with an almost uniform mesh, then the method becomes second-order accurate in smooth regions. Corollary 4.2 shows that when the diffusion parameter $\varepsilon$ is relatively small, the method is second-order accurate on any general tensor product mesh, away from any layers. Nevertheless, this $l_{2}$ seminorm is of course not strong enough to exclude checkerboard oscillations from the computed solution.

\section{ACKNowledgement}

We thank the referee for a meticulous reading of this paper.

\section{REFERENCES}

1. I. Babuška and J. Osborn, Analysis of finite element methods for second order boundary value problems using mesh dependent norms, Numer. Math. 34 (1980), 41 - 62. MR 81g:65143

2. H. Baumert, P. Braun, E. Glos, W. Müller and G. Stoyan, Modelling and computation of water quality problems in river networks, Lecture Notes in Control and Information Sciences, vol. 23, Springer, Berlin, 1981, pp. $482-491$.

3. W. Eckhaus and E. M. de Jager, Asymptotic solutions of singular perturbation problems for linear differential equations of elliptic type, Arch. Rational Mech. Anal. 23 (1966), 26 - 86. MR 34:6283

4. R. E. Ewing, The mathematics of reservoir simulation, SIAM, Philadelphia, 1983. MR 85g:76027

5. S.-Y. Hahn, An 'upwind' finite element method for electromagnetic field problems in moving media, Intern. J. Numer. Methods Engrg. 24 (1987), 2071 - 2086. 
6. M. Jakob, Heat transfer, Wiley, New York, 1959.

7. O. A. Ladyženskaja, V. A. Solonnikov and N. N. Ural'ceva, Linear and quasi-linear equations of parabolic type, Translations of Mathematical Monographs, Vol. 23, American Math. Soc., Providence, R.I., 1968. MR 39:3159b

8. J. A. Mackenzie and K. W. Morton, Finite volume solutions for convection-diffusion test problems, Math. Comp. 60 (1993), 189 - 220. MR 93d:76065

9. K. W. Morton and M. Stynes, An analysis of the cell vertex method, Math. Modél. Anal. Numér. 28 (1994), 699 - 724. MR 95h:65072

10. K. W. Morton and E. Süli, Finite volume methods and their analysis, IMA J. Numer. Anal. 11 (1991), 241 - 260. MR 93e:65145

11. A. H. Nayfeh, Perturbation methods, Wiley, New York, 1973. MR 53:8588

12. R. E. O'Malley, Singular perturbation methods for ordinary differential equations, SpringerVerlag, New York, 1991. MR 92i:34071

13. D. W. Peaceman and H. H. Rachford, Numerical calculation of multi-dimensional miscible displacement, Soc. Petroleum Engrg. J. 24 (1962), 327 - 338.

14. H. S. Price and R. S. Varga, Error bounds for semidiscrete Galerkin approximations of parabolic problems with applications to petroleum reservoir mechanics, in Numerical Solution of Field Problems in Continuum Physics, SIAM-AMS Proc., Vol. 2, 1970, pp. 74 - 94. MR 42:1358

15. E. Süli, The accuracy of finite volume methods on distorted partitions, in The Mathematics of Finite Elements and Applications (J. R. Whiteman, ed.), VII MAFELAP 1990, Academic Press, New York, 1991, pp. 253 - 260. MR 92i:65171

16. - The accuracy of cell vertex finite volume methods on quadrilateral meshes, Math. Comp. 59 (1992), 359 - 382. MR 93a:65158

17. M. Van Dyke, Perturbation methods in fluid mechanics, Parabolic Press, Stanford, CA, 1975. MR 54:4315

18. M. I. Vishik and L. A. Lyusternik, Regular degeneration and boundary layer for linear differential equations with a small parameter, Uspekhi Mat. Nauk. 12 (1957), 3 - $122=$ Amer. Math. Soc. Transl., Ser. 2, 20 (1962), pp. 239 - 364. MR 25:322

Department of Mathematics, University College, Cork, Ireland

E-mail address: stynes@ucc.ie 